\title{
Synthesis of Modified Silica Aerogel Nanoparticles for Remediation of Vietnamese Crude Oil Spilled on Water
}

\author{
Hong K. D. Nguyen, *,a Phuong T. Hoang ${ }^{a}$ and Ngo T. Dinh ${ }^{a}$ \\ ${ }^{a}$ School of Chemical Engineering, Hanoi University of Science and Technology, \\ No. 1 Dai Co Viet Street, Hai Ba Trung District, 10000 Hanoi, Vietnam
}

\begin{abstract}
New application of modified silica aerogel nanoparticles was carried out for adsorption of Vietnamese spilled crude oil obtained from Bach-Ho field. The aerogel was synthesized by atmospheric drying method without critical extraction procedure in liquefied $\mathrm{CO}_{2}$ followed by surface modification using polydimethylsiloxane precursor in $n$-hexane solvent. The synthesis mixture contained precise amounts of water, tetraethyl orthosilicate (TEOS), ethanol and $\mathrm{HCl}$ with volume ratios of 2:1:4:0.0003, respectively. The modification was established by the mixing of the as-synthesized aerogel and polydimethylsiloxane followed by calcination at $350{ }^{\circ} \mathrm{C}$ for $4 \mathrm{~h}$ obtaining the surface modified aerogel. The modified aerogel was applied in the adsorption of the spilled crude oil in mild temperature and atmospheric pressure. Some techniques such as X-ray diffraction (XRD), transmission electron microscopy (TEM), Brunauer-Emmett-Teller surface area analysis (BET) and Fourier transform infrared spectroscopy (FTIR) were used for characterizing the materials.
\end{abstract}

Keywords: aerogel, TEOS, modification, nanoparticle, silica

\section{Introduction}

Silica aerogel is the most common type of aerogel, and the most extensively studied and used. It is silica-based and could be derived from silica gel or by a modified Stober process. ${ }^{1}$ It has many unique properties such as high specific surface area, high porosity, ultra-low density, high thermal insulation value, low dielectric constant $(\mathrm{k}=1.0-2.0)$ and low index of refraction (ca. 1.05). ${ }^{1,2-4}$ In 1931, Kistler $^{5}$ used a process known as supercritical drying, which avoids a direct phase change to synthesize the first silica aerogel. The process was typically done by exchanging the initial aqueous pore liquid for a $\mathrm{CO}_{2}$-miscible liquid such as ethanol or acetone, then onto liquid carbon dioxide and then bringing the carbon dioxide above its critical point. ${ }^{1}$ Up to now, many processes were invented and applied to produce silica aerogels mainly focusing at different treatment of the drying process. There were methods such as supercritical drying, ambient pressure drying and freeze drying. The supercritical drying method included two approaches established at high and low temperatures. The high temperature treatment was firstly applied by Kistler ${ }^{5}$ in 1931, and the low temperature one was developed by

*e-mail: dieuhong_bk@yahoo.com, dieuhongprof@gmail.com
Tewari et al. ${ }^{6}$ in 1985 . Actually, in any way, the supercritical drying requires many unique equipment and procedures because of its extremely high pressure of the critical $\mathrm{CO}_{2}$ solvent. ${ }^{1,4,7-12}$

The drying method was freeze-drying, where the phase boundary between the liquid and gas phase did not exist and thus the capillary pressure does not play an important role. However, the freeze drying contained many disadvantages including the too long aging period, and in some situations, the aerogel network could be destroyed by crystallization of the solvent in the pores. ${ }^{1}$ The ambient pressure drying method offered great promise to lower costs for aerogel production and thus represented an important consideration for the future development of these materials. ${ }^{2}$ Therefore, the ambient pressure drying method was applied in this report with high performance of the silica aerogel nanoparticle synthesis. The aerogel was also modified with polydimethylsiloxane, which had much lower price than other precursors including dimethyldichlorosilane (DMDCS), hexamethyldisiloxane (HMDSO), phenyltrimethoxysilane (PTMS), and hexamethyldisilazane (HMDZ). ${ }^{8,12-15}$ The modification increased the hydrophobicity of the aerogel, which strengthened its ability in the adsorption of spilled crude oil. 
For the purposes of oil adsorption, especially oil spill remediation, the adsorption capacity and the hydrophobicity of the used materials were the most important characteristics improving the performance of the whole process. As well known, oil spills on freshwater could cause serious environmental and economic impacts, as they could influence the aquatic and nonaquatic life..$^{13,14,16,17}$ Furthermore, the chemical properties of the crude oil were strongly dependent on its origin and sources. Accordingly, its behavior in the water body was complex and cannot be easily predicted. For instance, some constituents of crude oil were noted for their tendency to float on water and vaporize; while others preferred binding to solid surfaces and making hard to separate the emulsion. ${ }^{12,13}$ Some remediation methods were applied for oil separation or gathering including bioremediation, controlled burning, skimming, solidification and by centrifugation. ${ }^{15}$ However, these methods have proven to be expensive, time-consuming and/or ineffective in meeting the strict environmental regulations. The adsorption process could be considered as one of the most significant method for the purpose. Different types of adsorbents have been widely used for water treatment and recyclability, such as activated carbon, copolymers, organoclays, zeolite and resins, ${ }^{12,16,17}$ but their oil adsorption capacity was not as high as expected. Therefore, the aerogel with high surface area, hydrophobicity and ultra-light density could greatly contribute to the sector.

For the whole mentioned reasons, our report provided some new aspects for solving these problems including preparation of the silica aerogel nanoparticle using ambient pressure drying method, surface modification of the as-synthesized aerogel, and application of the modified aerogel in the adsorption of the spilled Vietnamese Bach-Ho crude oil.

\section{Experimental}

\section{Chemicals}

Tetraethyl orthosilicate (TEOS), isopropanol, $n$-hexane, $25 \% \mathrm{NH}_{4} \mathrm{OH}$ solution, ethanol, $36.5 \% \mathrm{HCl}$ solution and polydimethylsiloxane were purchased from Merck and used without any further purification. Distilled water was produced in the laboratory.

Preparation and surface modification of silica aerogel nanoparticles

\section{Synthesis of silica aerogel nanoparticles}

The silica aerogel nanoparticles were prepared through sol-gel process followed by ambient pressure drying method. The procedure was as follows: many components such as distilled water, TEOS, ethanol and $\mathrm{HCl}$ solution were mixed together with volume ratios of 2:1:4:0.0003, respectively, in which the amount of water was $0.0448 \mathrm{~mol}$. The mixture was then supplied with more amount of ethanol, water and $\mathrm{NH}_{4} \mathrm{OH}$ solution with volume ratios of 7:3.5:0.002 (amount of ethanol was $0.0514 \mathrm{~mol}$ ) followed by vigorous stirring until the gel was formed in a good transparency. The gel was then transferred into $10 \mathrm{~mL}$ graduated volumetric cylinders for about one week at room temperature. The prepared samples after one week of aging were soaked with $n$-hexane before pouring into bottles filled with $100 \mathrm{~mL}$ isopropanol, then kept for $20 \mathrm{~h}$. A drying process was then established at $55^{\circ} \mathrm{C}$ for about $20 \mathrm{~h}$ at ambient pressure obtaining silica aerogel nanoparticles.

\section{Surface modification of silica aerogel nanoparticles}

A mixture including $1 \mathrm{~g}$ of silica aerogel and $0.2 \mathrm{~g}$ of polydimethylsiloxane was prepared, then it was homogeneously diffused in $50 \mathrm{~mL} n$-hexane under vigorous stirring at ambient temperature. The process was done until completely evaporating of $n$-hexane solvent. The white solid was then dried at $100{ }^{\circ} \mathrm{C}$ for $5 \mathrm{~h}$ and calcined at $350{ }^{\circ} \mathrm{C}$ for $4 \mathrm{~h}$ obtaining the modified silica aerogel nanoparticles.

Adsorption of spilled crude oil on modified silica aerogel nanoparticles

The oil adsorption of the spilled oil on the modified silica aerogel nanoparticles was established by a method given in a previous paper $^{18}$ using oil polluted water (simulation of the oil polluted water from Bach Ho oil field, Vietnam) as adsorption environment. The previous paper reported the adsorption on silica nanoparticles, while this paper tested the adsorption capacity on the modified aerogel. Various time values (for example, after 3, 5, 10, $15,30,60$ and $120 \mathrm{~min}$ of adsorption) were investigated for the adsorption at fixed temperature of $30^{\circ} \mathrm{C}$.

After a certain period of time, the contaminated aerogel was separated from the water. The adsorption capacity of the aerogel was determined by dividing the mass of the adsorbed oil to that of the used aerogel. The total amount of the adsorbed oil was calculated according to the given report. ${ }^{13}$

\section{Characterizations}

Transmission electron microscopy (TEM) images were captured using field emission JEOL 1100; X-ray diffraction (XRD) patterns were recorded using D8 Advance (Bruker); 
Fourier transform infrared (FTIR) spectra were measured in Nicolet 6700 FT-IR Spectrometer. Brunauer-Emmett-Teller surface area (BET) method was measured on Micromeritics Gemini VII 2390 V1.02.

\section{Results and Discussion}

Amorphous property of silica aerogel nanoparticles before and after surface modification

The silica aerogel nanoparticles before and after surface modification were characterized by XRD method. Figure 1 described these patterns, which confirmed the presence of amorphous nanosilica with broad, smooth background and no distinctive and clear peak. The XRD patterns were also not too different from that of the nanosilica ${ }^{18}$ with a peak at ca. $22^{\circ}$ leading to the confirmation that the aerogel was mostly amorphous existence.

The difference between the patterns of the silica aerogel nanoparticles before and after surface modification was

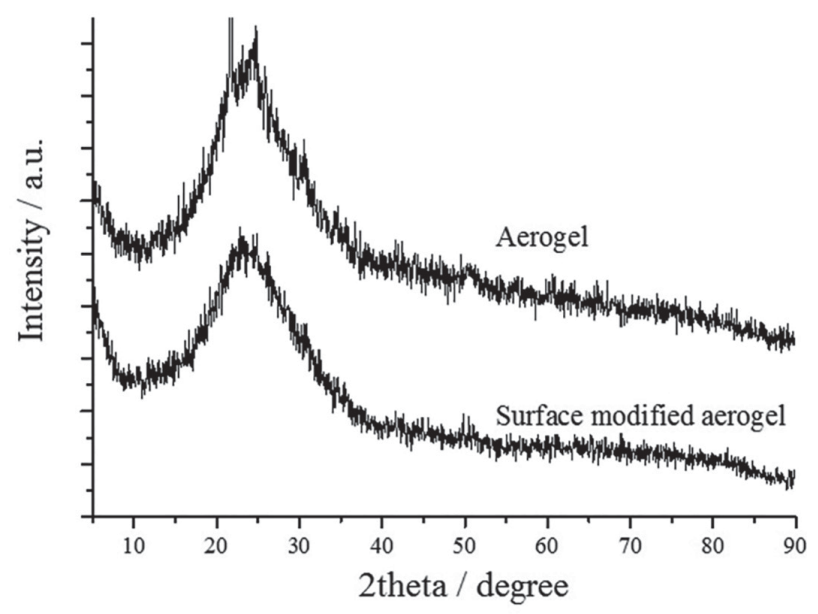

Figure 1. XRD patterns of the aerogel before and after surface modification. very small, demonstrating the stability of the aerogel framework during the modification and calcination. The reason for that could be assigned for the nature of the surface modification process. The polydimethylsiloxane would cover the surface of the silica aerogel nanoparticles in thin layers; therefore, the amorphous structure of the silica aerogel nanoparticles was maintained during the modification. On the other hand, the structure of the silica aerogel nanoparticles was not collapsed at the calcination after the modification, which also pointed out the good thermal stability of the material.

\section{Morphology of silica aerogel nanoparticles before and after surface modification}

TEM micrographs of the synthesized aerogel before and after surface modification were also exhibited in Figures 2 and 3, where the morphologies of these materials could be considered. It could be concluded that the silica aerogel nanoparticles exhibited a sponge-like microstructure. The spherical silica nanoparticles formed a three-dimensional network containing homogeneous pores (size in the range of 10-15 $\mathrm{nm}$ ). The particle sizes were estimated in the range of a few tens nanometers, clearly proving the formation of microparticle system.

By observing the surface of the aerogel before and after surface modification, the TEM images exhibited the presence of small dot areas that were darker than the surrounding ones. These suggested the presence of nucleation seeds or crystal-like seed. The dots were well distributed before and after the surface modification, demonstrating that the aerogel possessed high thermostability property even when being calcinated at $350{ }^{\circ} \mathrm{C}$ for a long period.

The same morphologies between the aerogel before and after modification could be explained by the fact that the modification only connected the modifier

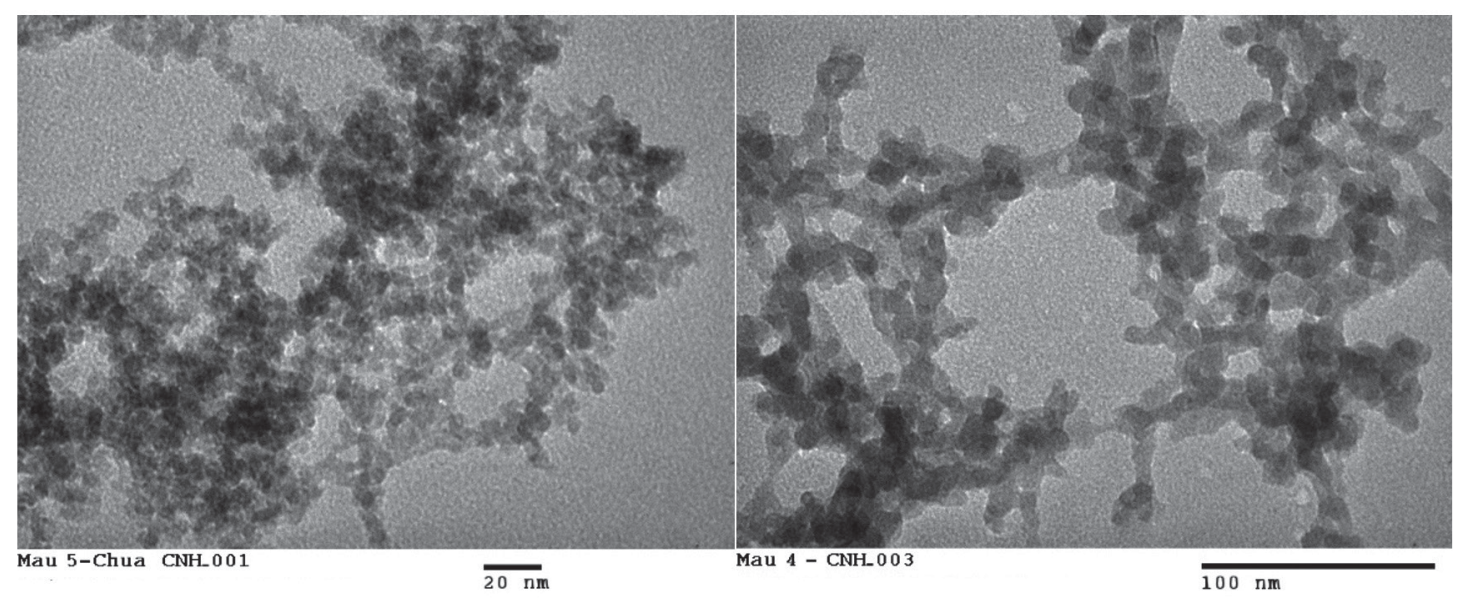

Figure 2. TEM images of silica aerogel nanoparticles. 


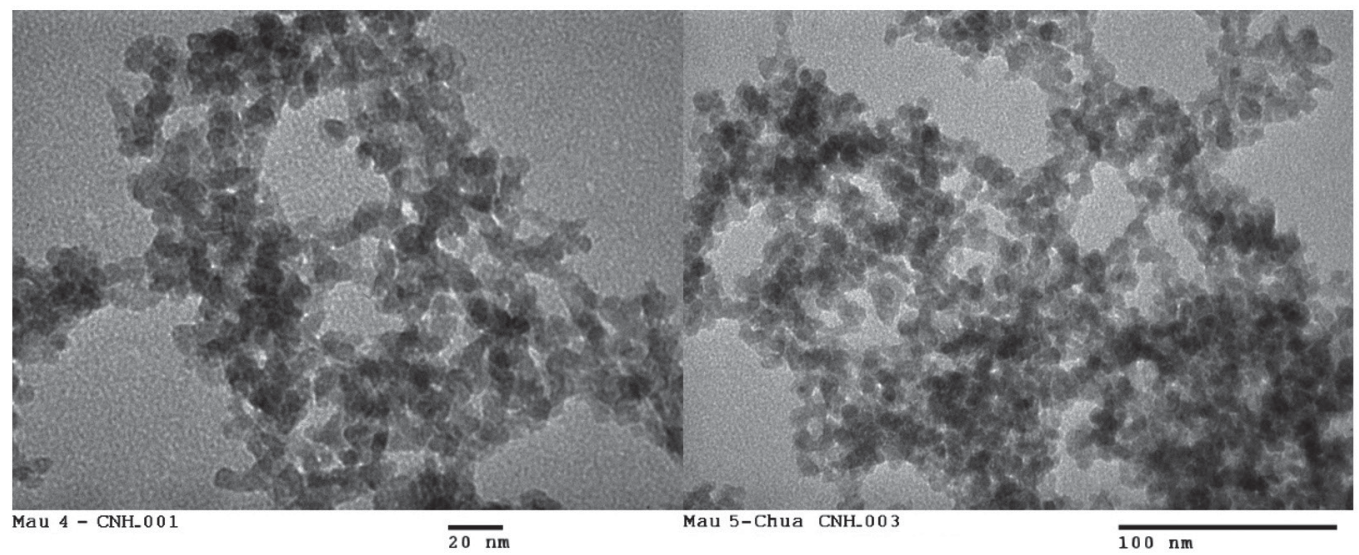

Figure 3. TEM images of surface modified silica aerogel nanoparticles.

polydimethylsiloxane and the aerogel without any changes in the structure of the silica frameworks. The modifier played as a provider of $-\mathrm{CH}_{3}$ groups for the hydrophilic $\mathrm{Si}-\mathrm{OH}$ groups of the aerogel to help them becoming the hydrophobic ones.

Different surface groups of silica aerogel nanoparticles before and after the modification

FTIR was applied to investigate the differences of functional groups between the aerogel and modified aerogel. Figure 4 described the FTIR spectra of these silica aerogel nanoparticles.

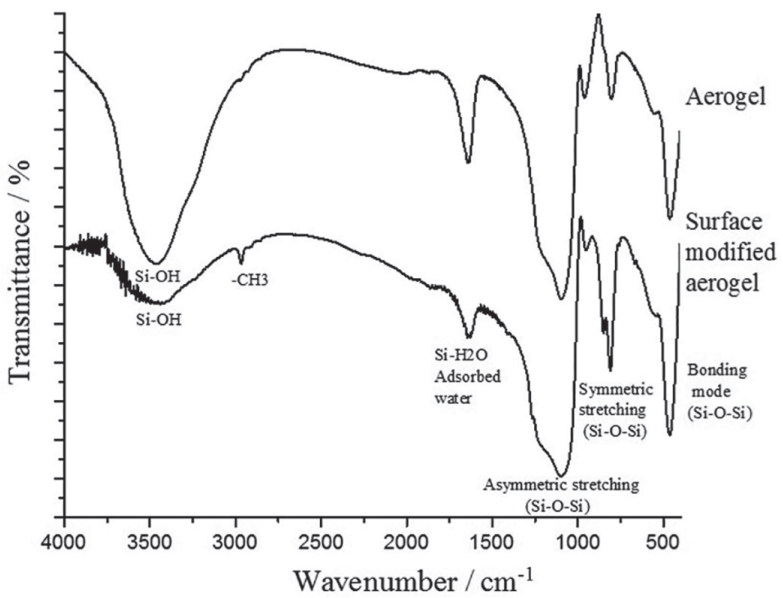

Figure 4. FTIR spectra of the silica aerogel nanoparticles before and after surface modification.

The FTIR spectra showed that the silica aerogel nanoparticles before and after surface modification both had peaks at ca. $3400-3500 \mathrm{~cm}^{-1}$ assigned for the stretching vibration of the hydroxyl groups typical for the silanol functions ( $\mathrm{Si}-\mathrm{OH})$.

There were also the appearances of the strong peaks at ca. $465 \mathrm{~cm}^{-1}$ assigned for the bending vibration of the
$\mathrm{O}-\mathrm{Si}-\mathrm{O}$ and $\mathrm{Si}-\mathrm{O}-\mathrm{Si}$ functional groups, at $1150 \mathrm{~cm}^{-1}$ assigned for the symmetric streching of $\mathrm{Si}-\mathrm{O}-\mathrm{Si}$ linkages, and peaks at $1750 \mathrm{~cm}^{-1}$ assigned for the asymmetric stretching of the $\mathrm{Si}-\mathrm{O}-\mathrm{Si}$ systems and the -OH hydrogen connection of the adsorbed wate. These vibrations clearly proved the formation of the silica nanoparticles.

There was a small difference between the FTIR spectra of the two materials that appeared at around $3050 \mathrm{~cm}^{-1}$ related to the stretching vibration of the $\mathrm{CH}_{3}$ groups in the surface modified aerogel (Figure 5). This was a rigid evidence that positively confirmed the successful modification of the silica aerogel by using polydimethylsiloxane. As a consequence, the modification provided the aerogel a high hydrophobic property. This result could be observed in the peak at ca. $3400 \mathrm{~cm}^{-1}$ : the peak intensity of the $-\mathrm{OH}$ groups in the aerogel after modification was much lower than that of the aerogel before modification. Some specification of the silica aerogel nanoparticles before and after surface modification were also collected in Table 1.<smiles>C[Si](C)(C)O[Si](C)(C)O[Si](C)(C)C</smiles>

Figure 5. Proposed structured of surface modified silica aerogel nanoparticles.

The specification showed that the aerogel before and after surface modification exhibited distinct properties, especially relating to the water solubility. The extreme decrease in water solubility through the surface modification was strictly caused by the increase in the hydrophobic property of the material. By this phenomenon, the aerogel could be applied in the oil adsorption without loss in the adsorbent. 
Table 1. Specifications of the silica aerogel nanoparticles before and after surface modification

\begin{tabular}{lcccc}
\hline No. & Property & ASTM method & Aerogel & Surface modified aerogel \\
\hline 1 & apparent density & D 1895 & 0.02292 & 0.0309 \\
2 & tensile strength / kPa & D 638 & 14.5 & 15.0 \\
3 & water solubility / \% & D 5907 & 4.250 & 0.005 \\
4 & index of refraction & D 542 & 1.01 & 1.01 \\
5 & linear thermal expansion & E 831 & $3.0 \times 10^{-6}$ & $3.0 \times 10^{-6}$ \\
\hline
\end{tabular}

Texture properties of the surface modified silica aerogel nanoparticles

The BET surface area of the surface modified silica aerogel nanoparticles reached $574.3782 \mathrm{~m}^{2} \mathrm{~g}^{-1}$, demonstrating its high porosity. This property would greatly enhance the oil diffusion into the porous system of the aerogel. The adsorption-desorption isotherm of the aerogel was plotted in Figure 6.

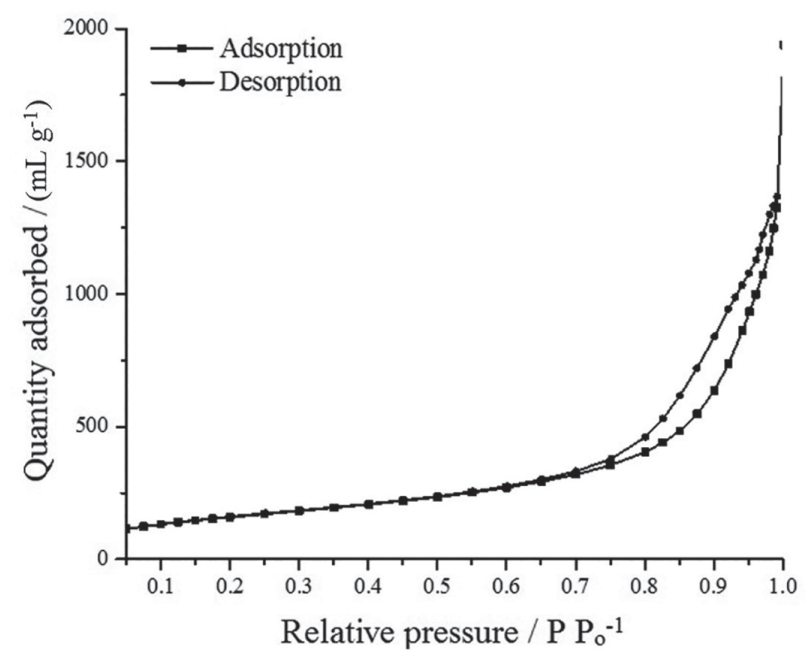

Figure 6. Adsorption-desorption isotherm of the silica aerogel nanoparticles.

The isotherm also pointed out that a mesoporous system existed in the material with hysteresis loop appearing at high relative pressure. The mesoporosity also greatly strengthened the oil adsorption capacity of the aerogel. The pore distribution due to pore area and volume were also plotted in Figures 7 and 8 .

The pore volume distribution of the aerogel averaged at ca. $135 \AA$ and a density of $2.026801 \mathrm{~cm}^{3} \mathrm{~g}^{-1}$. This high value was because of the concentration of the large pore width, which was convenient for the storage of the adsorbed oil.

The distribution of the surface area of the aerogel mainly concentrated at ca. $130 \AA$ and ca. $200 \AA$ A , corresponding to the mesoporous system proven by the isotherm. This value was larger than that of the normal ordered mesoporous material because of the disordered distribution of the

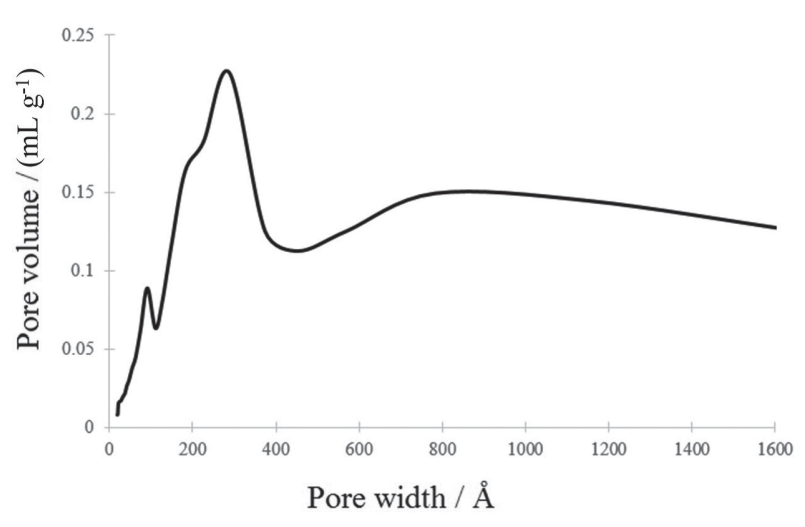

Figure 7. Pore distribution by BJH method due to pore volume.

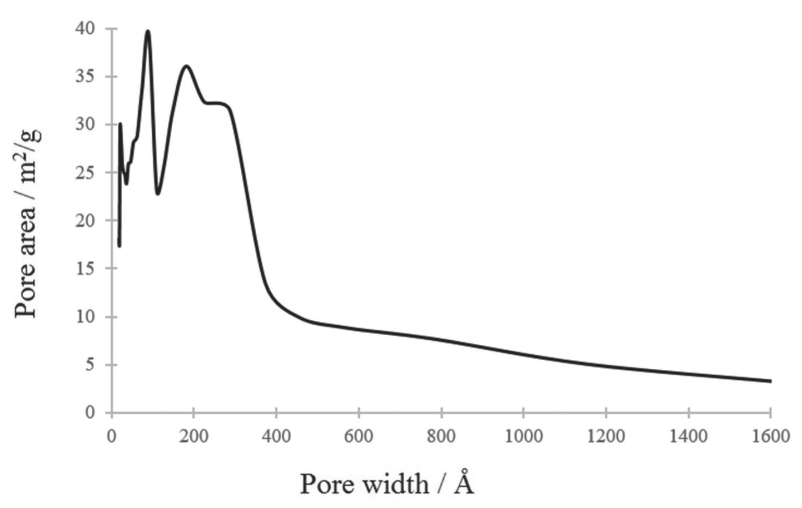

Figure 8. Pore distribution by BJH method due to pore area.

mesopores. The large pore width, on the other hand, was convenient for the oil diffusion along with the pore channels.

Oil adsorption capacity of the surface modified silica aerogel nanoparticles

The testing for oil adsorption of the silica aerogel nanoparticles was established using Bach Ho crude oil with some properties listed in Table 2. These specifications were also described in an elsewhere paper. ${ }^{18}$

The adsorption testing with Bach Ho crude oil using the two kind of material was exhibited in Figures 9 and 10. The Bach Ho crude oil was spread on the surface of the water before applying the adsorption process. 
Table 2. Bach Ho crude oil properties

\begin{tabular}{lccc}
\hline No. & Property & $\begin{array}{c}\text { ASTM } \\
\text { method }\end{array}$ & Value \\
\hline 1 & density, $20^{\circ} \mathrm{C}$ & D 3505 & 0.8423 \\
2 & melting point / ${ }^{\circ} \mathrm{C}$ & D 87 & 22.35 \\
3 & average molecule mass / $\left(\mathrm{g} \mathrm{mol}^{-1}\right)$ & D 6579 & 250.9 \\
4 & kinematic viscosity, $50^{\circ} \mathrm{C} / \mathrm{cSt}$ & D 445 & 12.33 \\
5 & sulfur content / \% & D 4294 & 0.0402 \\
6 & paraffin content / \% & D 5443 & 22.9 \\
7 & asphalt content / \% & D 6560 & 4.02 \\
\hline
\end{tabular}

The results obtained from Figure 9 showed that the maximum adsorption capacity of the silica aerogel nanoparticles before modification was 15.38 , which was higher than that of nanosilica, but lower than that of surface modified nanosilica published in another report. ${ }^{18}$ The reason for its low adsorption capacity was related to its low hydrophobicity with a large amount of water dissolved material.

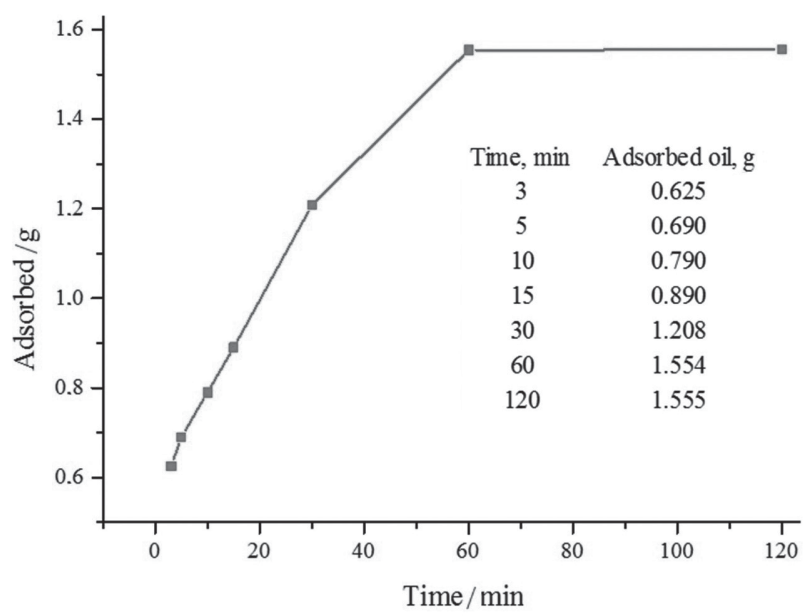

Figure 9. Oil adsorption at different times using silica aerogel nanoparticles.

The oil adsorption capacity raised from 15.38 to 153.47 after surface modification, clearly demonstrating the very good ability of the silica aerogel nanoparticles in the oil recovery (Figure 10). The oil adsorption capacity increased from 15.38 to 153.47 after surface modification, clearly demonstrating the good ability of the silica aerogel nanoparticles in the oil recovery (Figure 10). The oil adsorption capacity of the surface modified silica aerogel nanoparticles was also higher than that of the nanosilica materials in the mentioned paper. ${ }^{13}$

\section{Conclusions}

The silica aerogel nanoparticles were sucesfully

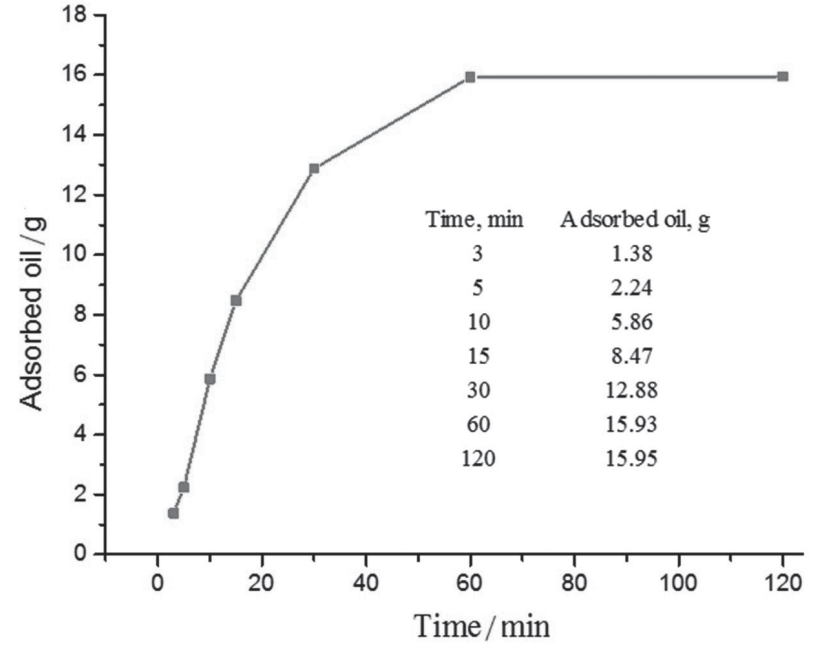

Figure 10. Oil adsorption at different times using surface modified silica aerogel nanoparticles.

prepared and modified by sol-gel method and by using polydimethylsiloxane, respectively. The modified aerogel exhibited high hydrophobicity because of functionalizing of the aerogel nanoparticles by $\mathrm{CH}_{3}$ groups, high surface area and large pore width, which considerable enhanced diffusion of the adsorbent into these channels.

It was investigated the adsorption by using Vietnamese crude oil on the modified aerogel. Its adsorption capacity was compared to that of the other material such as normal silica aerogel nanoparticles, nanosilica and surface modified nanosilica. The results confirmed that the modified silica aerogel nanoparticles had the greatest capacity in the spilled oil remediation over the water.

\section{References}

1. Azadeh, T.; Marzieh, H.; Vahid, M.; Mater. Res. Bull. 2012, 47, 2584.

2. Fricke, J.; J. Non-Cryst. Solids 1988, 100, 169.

3. Carraher Jr., C. E.; Polym. News 2005, 30, 386.

4. Schultz, J. M.; Jensen, K. I.; Kristiansen, F. H.; Sol. Energy Mater. Sol. Cells 2005, 89, 275.

5. Kistler, S. S.; Nature 1931, 127, 741.

6. Tewari, P. H.; Hunt, A. J.; Lofftus, K. D.; Mater. Lett. 1985, 3, 363.

7. Maury, S.; Buisson, P.; Pierre, A. C.; J. Mol. Catal. B: Enzym. 2002, 19, 269.

8. Aravind, P. R.; Shajesh, P.; Soraru, G. D.; Warrier, K. G. K.; J. Sol-Gel Sci. Technol. 2010, 54, 105.

9. Schmidt, M.; Schwertfeger F.; J. Non-Cryst. Solids 1998, 225, 364.

10. Gibiat, V.; Lefeuvre, O.; Woignier, T.; Pelous, J.; Phalippou, J.; J. Non-Cryst. Solids 1995, 186, 244.

11. Chwastowski, J.; Figiel, J.; Kotarba, A.; Olkiewicz, K.; Suszycki, L.; Nucl. Instrum. Methods Phys. Res., Sect. A 2003, 504, 222. 
12. Woignier, T. T.; Reynes, J.; Phalippou, J.; Dussossoy, J. L.; Jacquet-Francillon, N. N.; J. Non-Cryst. Solids 1998, 225, 353.

13. Fingas, M.; Fieldhouse, B.; Mar. Pollut. Bull. 2003, 47, 369.

14. Elkins, P.; Vanner, R.; Firebrace, J.; J. Cleaner Prod. 2007, 15, 1302.

15. Ahmaun, F.; Pendashteh, A.; Chuah, L.; Radiah, D.; Siavash, S.; Zainal, Z.; J. Hazard. Mater. 2009, 170, 530.

16. Yang, T. R.; Adsorbents: Fundamentals and Applications; Wiley-Interscience: Hoboken, NJ, 2003.
17. Harper, R. T.; Johnston, J. H.; Wiseman, N.; U.S. Patent No. 5,200,165 1993.

18. Hoang, P. T.; Nguyen, T. T.; Dinh, N. T.; Vietnam. J. Adsorpt. Catal. (in Vietnamese) 2016, 5, 75.

Submitted: July 20, 2017

Published online: March 13, 2018 